\title{
Compétences sociales
}

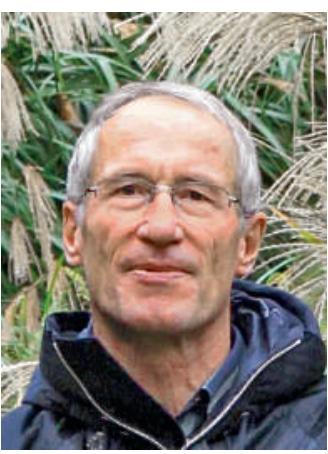

Erhard Taverna

Pas une offre d'emploi qui ne mentionne le terme de «compétences sociales» ou «softskills» et «qualités de coaching» pour parler français. Ce qui est exigé par l'économie doit être enseigné dans les universités. Un concept que Pestalozzi décrivait encore en termes de «tête, cour et main», soit l'intellect, l'affectif et les connaissances, a été rebaptisé en «Social credit points» selon le modèle du European Credit Transfer System (ECTS). On entend par là les points supplémentaires à acquérir suivant une charge de travail définie et qui permettent d'obtenir une plus-value sociale accumulable et transférable dans toute l'Europe.

A l'Université de Lucerne, il est obligatoire jusqu'à l'obtention du bachelor d'acquérir 4 points valant 90 heures de travail. On vise ainsi à développer les compétences qui seront ensuite utiles dans la vie professionnelle à savoir l'engagement, l'esprit d'équipe, la coopération et les qualités de dirigeant. Selon les indications fournies par la Faculté des sciences sociales, ce système a pour but d'intégrer progressivement les étudiants dans la gestion autonome de l'université au travers de cercles de lecture, de tutorats liés à des manifestations, de participation à des commissions internes

\section{Peut-être que l'encouragement précoce, la sélection des élèves particulièrement doués et le stress des cours particuliers ont occasionné des dommages collatéraux.}

ou de stages structurés dans le cadre des études de master. Les travaux de nettoyage, l'aide en cuisine ou à la cantine ne figurent toutefois pas au programme. La Faculté de droit propose des stages rémunérés et pour la Faculté de théologie, il suffit que des personnes dans le besoin bénéficient du travail des futurs théologiens.

La définition des compétences sociales varie selon le domaine d'activité. Ce qui est considéré comme une compétence dans un domaine peut être perçu comme une lacune dans un autre. Où d'aucuns exigent rigueur et détermination, d'autres demandent esprit d'équipe, empathie et courage civique. L'attribution des points doit comprendre plus d'activités pratiques et ainsi favoriser le développement personnel des étudiants. Les universités de Berne, Zurich et Bâle ont renoncé à rendre ces activités obligatoires. Elles préfèrent une approche intégrative par l'apprentissage de l'esprit d'équipe et de la gestion des conflits au travers de séminaires et de tutorats. Les écoles étrangères attribuent également des points aux activités extra-scolaires comme la participation à l'équipe de sport de l'école, à des festivals de musique et à des symposiums.
Et même si ces termes demeurent un peu flous, on parle d'ores et déjà de standardisation. Les «Social credits» seraient des outils de promotion intéressants qu'il conviendrait d'introduire au niveau secondaire déjà. Les psychologues férus de tests veulent obtenir des résultats mesurables afin de pouvoir comparer les écoles supérieures entres elles. Ils veulent établir une classification, comme cela se fait déjà avec les étudiants dont les résultats sont comparés à ceux d'au moins 50 autres par le biais d'une évaluation statistique et de pourcentages.

Malgré les bonnes intentions et les souhaits d'opérationnalisation, on peut quand même se demander quel est le sens d'une telle démarche. Avant d'introduire les tests d'aptitude en médecine, on envoyait les étudiants en stage dans un hôpital ou dans des infirmeries ou on leur demandait d'autres prestations de soins ou sociales. Ces étudiants, hommes et femmes confondus, devaient terminer leurs études en disposant de compétences professionnelles solides et de convictions éthiques.

A l'époque de la guerre froide, le service militaire était considéré comme une bonne école pour apprendre à diriger. Il servait de modèle en matière de discipline, d'endurance et de développement des facultés de communication. Ce que les scouts et ensuite le service militaire offraient gratuitement fut longtemps un exercice obligatoire pour les cadres d'entreprises. Par des cours onéreux leur faisant construire des ponts suspendus ou faire des bivouacs, on cherchait à renforcer leur esprit d'équipe. De nombreuses méthodes permettent d'acquérir des compétences sociales, elles suivent la mode et les idées novatrices fort rentables des instituts de formation continue tant publics que privés.

Se peut-il que les écoles supérieures se qualifient elles-mêmes sans le vouloir? Par un système qui exige autant de ses étudiants que leurs compétences sociales s'atrophient? Peut-être que celles-ci ont déjà fondu durant les années d'école obligatoire. Peut-être que l'encouragement précoce, la sélection des élèves particulièrement doués et le stress des cours particuliers ont occasionné des dommages collatéraux. Si on feuillète les multiples brochures posées sur les tables de la cafétéria de l'université, on remarquera qu'elles ne parlent que choix de carrière, entretien d'embauche et sélection. Des termes qui font perdre leur sens aux compétences sociales et aux autres formes d'éthique, car elles ne concerneraient pas le marché réel. Les qualités exigées aujourd'hui sont la compétitivité, la concurrence, la capacité d'adaptation, l'opportunisme, la rapidité, la flexibilité, l'esprit tactique, la rage de vaincre et le narcissisme. Mais cela bien sûr uniquement à un dosage équilibré favorisant la réussite sociale.

Erhard Taverna 